\title{
Indestructible colourings and rainbow Ramsey theorems
}

by

\author{
Lajos Soukup (Budapest)
}

\begin{abstract}
We show that if a colouring $c$ establishes $\omega_{2} \nrightarrow\left[\left(\omega_{1}: \omega\right)\right]^{2}$ then $c$ establishes this negative partition relation in each Cohen-generic extension of the ground model, i.e. this property of $c$ is Cohen-indestructible. This result yields a negative answer to a question of Erdős and Hajnal: it is consistent that GCH holds and there is a colouring $c:\left[\omega_{2}\right]^{2} \rightarrow 2$ establishing $\omega_{2} \nrightarrow\left[\left(\omega_{1}: \omega\right)\right]_{2}$ such that some colouring $g:\left[\omega_{1}\right]^{2} \rightarrow 2$ does not embed into $c$.

It is also consistent that $2^{\omega_{1}}$ is arbitrarily large, and there is a function $g$ establishing $2^{\omega_{1}} \nrightarrow\left[\left(\omega_{1}, \omega_{2}\right)\right]_{\omega_{1}}$ but there is no uncountable $g$-rainbow subset of $2^{\omega_{1}}$.

We also show that if GCH holds then for each $k \in \omega$ there is a $k$-bounded colouring $f:\left[\omega_{1}\right]^{2} \rightarrow \omega_{1}$ and there are two c.c.c. posets $\mathcal{P}$ and $\mathcal{Q}$ such that $V^{\mathcal{P}} \models f$ c.c.c.-indestructibly establishes $\omega_{1} \nrightarrow^{*}\left[\left(\omega_{1} ; \omega_{1}\right)\right]_{k \text {-bdd }}$,

but

$V^{\mathcal{Q}} \models \omega_{1}$ is the union of countably many $f$-rainbow sets.

1. Introduction. Anti Ramsey (polychromatic Ramsey, rainbow Ramsey) theory deals with the following kind of problems: given a colouring $f$ of certain subsets of a set $X$, can you find a large subset $Y$ of $X$ such that $f$ is inhomogeneous (e.g. injective) on the coloured subsets of $Y$ ? Obviously, to get positive results we should have some assumption concerning the colouring $f$. In the first part of the paper we will assume that

- $f$ establishes some negative partition relation,
\end{abstract}

i.e. there are no large $f$-homogeneous sets, and we will try

- to get large $f$-inhomogeneous sets,

or, more generally,

2000 Mathematics Subject Classification: 03E02, 03E35, 03E50, 05D10.

Key words and phrases: anti Ramsey, rainbow Ramsey, polychromatic Ramsey, indestructible, forcing, partition relations, Martin's Axiom.

The preparation of this paper was supported by the Hungarian National Foundation for Scientific Research grant no. 61600 and 68262. 
- to show that $f$ is universal for certain colourings,

i.e. a certain class of colourings embeds into $f$. Let us recall that given two colourings $d:[A]^{2} \rightarrow \lambda$ and $f:[Y]^{2} \rightarrow \lambda$ we say that $d$ embeds into $f$, or $f$ realizes $d$ ( $d \Rightarrow f$, for short) iff there is a colour-preserving injection $\Phi: A \stackrel{1-1}{\longrightarrow} Y$, i.e.

$$
\left(\forall\{\zeta, \xi\} \in[A]^{2}\right) d(\zeta, \xi)=f(\Phi(\zeta), \Phi(\xi)) .
$$

To formulate our results we will use different kinds of "arrow" notation, so first we recall their definitions.

If $A$ and $B$ are two sets of ordinals let

$$
\begin{aligned}
& {[A, B]=\{\{\alpha, \beta\}: \alpha \in A, \beta \in B\},} \\
& {[A ; B]=\{\{\alpha, \beta\}: \alpha \in A, \beta \in B, \alpha<\beta\} .}
\end{aligned}
$$

Write $A \leq B$ iff $\sup A<\min B$. If $|A|=|B|$ write $A \triangleleft B$ iff $\operatorname{otp}(A \cap \alpha)<$ $\operatorname{otp}(B \cap \alpha)$ for each $\alpha \in A$. We now define some negative partition relation as follows.

Definition 1.1. Let $\kappa, \lambda, \mu$ and $\gamma$ be cardinals. We say that a function $f:[\kappa]^{2} \rightarrow \gamma$ establishes the negative partition relation

- $\kappa \nrightarrow[\lambda]_{\gamma}^{2}$ iff $\left(\forall A \in[\kappa]^{\lambda}\right) f^{\prime \prime}[A]^{2}=\gamma$,

- $\kappa \nrightarrow[(\mu, \lambda)]_{\gamma}$ iff $\left(\forall A \in[\kappa]^{\mu}\right)\left(\forall B \in[\kappa]^{\lambda}\right) f^{\prime \prime}[A, B]=\gamma$,

- $\kappa \nrightarrow[(\lambda ; \lambda)]_{\gamma}$ iff $\left(\forall A, B \in[\kappa]^{\lambda}\right)$ if $A \triangleleft B$ then $f^{\prime \prime}[A ; B]=\gamma$,

- $\kappa \nrightarrow[(\mu: \lambda)]_{\gamma}$ iff $\left(\forall A \in[\kappa]^{\mu}\right)\left(\forall B \in[\kappa]^{\lambda}\right)$ if $A<B$ then $f^{\prime \prime}[A, B]=\gamma$.

A negative partition relation holds iff there is a function establishing it.

If $\mu<\lambda \leq \kappa$ then clearly $\kappa \nrightarrow[(\mu: \lambda)]_{\gamma}$ implies $\kappa \nrightarrow[(\lambda ; \lambda)]_{\gamma}$ implies $\kappa \nrightarrow[(\lambda, \lambda)]_{\gamma}$ implies $\kappa \nrightarrow[\lambda]_{\gamma}^{2}$, moreover $f$ establishes $\kappa \nrightarrow[(\kappa ; \kappa)]_{\gamma}$ iff $f^{\prime \prime}[A ; B]=\gamma$ for each $A, B \in[\kappa]^{\kappa}$.

For a graph $G=\langle V, E\rangle$ define the function $\chi_{G}:[V]^{2} \rightarrow 2$ by the formula $E=\chi_{G}^{-1}\{1\}$. Clearly a graph $G$ is isomorphic to a spanned subgraph of a graph $H$ iff $\chi_{G} \Rightarrow \chi_{H}$, i.e. $\chi_{G}$ embeds into $\chi_{H}$.

Using this observation we can translate some results and problems of Erdős and Hajnal from the language of graphs and spanned subgraphs into the language of colourings and embeddings.

Erdős and Hajnal [5] observed that if a colouring $g:\left[\omega_{1}\right]^{2} \rightarrow 2$ establishes $\omega_{1} \nrightarrow\left[\left(\omega, \omega_{1}\right)\right]_{2}$ then $g$ is universal for countable "graphs", i.e., every function $h:[\omega]^{2} \rightarrow 2$ embeds into $g$. This result cannot be generalized to higher cardinals because of the following result of Shelah [10, Theorem 4.1]: It is consistent that GCH holds and there is a colouring $g:\left[\omega_{2}\right]^{2} \rightarrow 2$ which establishes $\omega_{2} \nrightarrow\left[\left(\omega_{1}, \omega_{2}\right)\right]_{2}$, but some colouring $h:\left[\omega_{1}\right]^{2} \rightarrow 2$ does not embed into $g$. 
Learning Shelah's result Erdős and Hajnal raised the following question in [6, Problem 6.b]: Assume that a colouring $g:\left[\omega_{2}\right]^{2} \rightarrow 2$ establishes $\omega_{2} \nrightarrow$ $\left(\omega_{1} \dot{+} \omega\right)_{2}^{2}$ (i.e. there is no $g$-monochromatic set of order type $\omega_{1} \dot{+} \omega$ ). Do all colourings $c:\left[\omega_{1}\right]^{2} \rightarrow 2$ embed into $g$ ?

We answer their question in the negative in Theorem 2.4. The proof is based on Theorem 2.2 which says that the property "g establishes $\omega_{2} \nrightarrow$ $\left[\left(\omega_{1}: \omega\right)\right]_{2}$ " is indestructible by adding an arbitrary number of Cohen reals to the ground model.

Given a colouring $f:[X]^{n} \rightarrow C$, a subset $P \subset X$ is called rainbow for $f$ (or $f$-rainbow) iff $f \uparrow[P]^{n}$ is one-to-one. We also answer another question of Hajnal, [9, Problem 4.1], in the negative in Theorem 2.6: it is consistent with GCH that there is a function $f$ which establishes $\omega_{2} \nrightarrow\left[\left(\omega_{1}: \omega\right)\right]_{\omega_{1}}$ such that there is no uncountable $f$-rainbow set.

In Theorem 2.8 we show that it is also consistent that $2^{\omega_{1}}$ is arbitrarily large and there is a function $g$ that establishes $2^{\omega_{1}} \nrightarrow\left[\left(\omega_{1}, \omega_{2}\right)\right]_{\omega_{1}}$ such that there is no uncountable $g$-rainbow set.

In the second part of the paper we deal with rainbow Ramsey theorems in which we have a different type of restriction concerning our colourings. Instead of establishing negative partition relations we assume that our colourings are "bounded": a function $f:[X]^{n} \rightarrow C$ is $\mu$-bounded iff $\left|f^{-1}\{c\}\right| \leq \mu$ for each $c \in C$.

To formulate our result we should recall one more "arrow" notation: $\lambda \rightarrow^{*}(\alpha)_{\kappa \text {-bdd }}^{n}$ holds iff for every $\kappa$-bounded colouring of $[\lambda]^{n}$ there is a rainbow set of order type $\alpha$.

We say that a function $f$ c.c.c.-indestructibly establishes the negative partition relation $\Phi \nrightarrow^{*} \Psi$ iff

$$
V^{P} \models f \text { establishes } \Phi \nrightarrow^{*} \Psi
$$

for each c.c.c. poset $P$.

Since $\omega_{1} \rightarrow(\alpha)_{2}^{2}$ holds for $\alpha<\omega_{1}$ by [4], and it was proved by Galvin [7] that

$$
\lambda \rightarrow(\alpha)_{k}^{n} \text { implies } \lambda \rightarrow^{*}(\alpha)_{k \text {-bdd }}^{n},
$$

we have $\omega_{1} \rightarrow^{*}(\alpha)_{2 \text {-bdd }}^{2}$ for $\alpha<\omega_{1}$. (A proof of $(*)$ can be found in [1], or see the proof of $(\star)$ just before Theorem 3.5 in the present paper.) Moreover, Galvin [7] showed that

Theorem. CH implies that $\omega_{1} \nrightarrow^{*}\left(\omega_{1}\right)_{2 \text {-bdd }}^{2}$.

(Lemma 3.3 also gives the result above.) On the other hand, Todorčević [12] proved that

Theorem. PFA implies that $\omega_{1} \rightarrow^{*}\left(\omega_{1}\right)_{2 \text {-bdd }}^{2}$. 
Abraham, Cummings and Smyth showed that $\mathrm{MA}_{\aleph_{1}}$ is not enough to get $\omega_{1} \rightarrow^{*}\left(\omega_{1}\right)_{2 \text {-bdd }}^{2}$. More precisely, they proved the following theorem:

THEOREM ([1, Theorem 3]). It is consistent that there is a function $c$ : $\left[\omega_{1}\right]^{2} \rightarrow \omega_{1}$ which c.c.c.-indestructibly establishes $\omega_{1} \nrightarrow^{*}\left(\omega_{1}\right)_{2-\text { bdd }}^{2}$.

They also showed that the property that $c$ establishes $\omega_{1} \nrightarrow^{*}\left(\omega_{1}\right)_{2 \text {-bdd }}^{2}$ is not automatically c.c.c.-indestructible:

Theorem $([1$, Theorem 4]). If CH holds and there is a Suslin tree then there is a function $c^{\prime}:\left[\omega_{1}\right]^{2} \rightarrow 2$ and a c.c.c. poset $P$ such that

(a) $c^{\prime}$ establishes $\omega_{1} \nrightarrow^{*}\left(\omega_{1}\right)_{2 \text {-bdd }}^{2}$,

(b) $V^{P} \models$ there is an uncountable $c^{\prime}$-rainbow set.

We say that the negative partition relation $\omega_{1} \nrightarrow^{*}\left[\left(\omega_{1} ; \omega_{1}\right)\right]_{\kappa \text {-bdd }}$ holds iff there is a $\kappa$-bounded colouring $c$ of $\left[\omega_{1}\right]^{2}$ such that for each $A, B \in\left[\omega_{1}\right]^{\omega_{1}}$ there is $\xi \in \operatorname{ran} c$ such that $|\{\{\alpha, \beta\} \in[A ; B]: c(\alpha, \beta)=\xi\}|=k$.

Clearly $\omega_{1} \nrightarrow^{*}\left[\left(\omega_{1} ; \omega_{1}\right)\right]_{2 \text {-bdd }}$ implies $\omega_{1} \nrightarrow^{*}\left(\omega_{1}\right)_{2 \text {-bdd }}^{2}$. We show that even the negative partition relation $\omega_{1} \nrightarrow^{*}\left[\left(\omega_{1} ; \omega_{1}\right)\right]_{k \text {-bdd }}$ is consistent with $\mathrm{MA}_{\aleph_{1}}$ for each $k \in \omega$.

Moreover, Abraham, Cummings and Smyth used two different functions in their theorems above. We show that a single function can play a double role.

THEOREM 1.2. If GCH holds then for each $k \in \omega$ there is a $k$-bounded colouring $f:\left[\omega_{1}\right]^{2} \rightarrow \omega_{1}$ and two c.c.c. posets $\mathcal{P}$ and $\mathcal{Q}$ such that $V^{\mathcal{P}} \models f$ c.c.c.-indestructibly establishes $\omega_{1} \nrightarrow^{*}\left[\left(\omega_{1} ; \omega_{1}\right)\right]_{k \text {-bdd }}$, but

$$
V^{\mathcal{Q}} \models \omega_{1} \text { is the union of countably many } f \text {-rainbow sets. }
$$

The following question, however, remained open.

Problem 1.3. Does $\omega_{1} \nrightarrow^{*}\left(\omega_{1}\right)_{2 \text {-bdd }}^{2}$ imply $\omega_{1} \nrightarrow^{*}\left[\left(\omega_{1} ; \omega_{1}\right)\right]_{2 \text {-bdd }}$ ?

2. On a problem of Erdős and Hajnal. Given two functions $f$ : $[X]^{2} \rightarrow C$ and $d:[Y]^{2} \rightarrow C$ we say that $d$ embeds into $f(d \Rightarrow f$, for short $)$, iff there is a one-to-one map $\Phi: Y \rightarrow X$ such that $d\left(y, y^{\prime}\right)=f\left(\Phi(y), \Phi\left(y^{\prime}\right)\right)$ for each $\left\{y, y^{\prime}\right\} \in[Y]^{2}$.

Hajnal [8] proved that it is consistent with GCH that there is a colouring establishing $\omega_{2} \nrightarrow\left(\omega_{1} \dot{+} \omega\right)_{2}^{2}$. It turns out that his argument gives the following stronger result:

Proposition 2.1. It is consistent that GCH holds and there is a function $f:\left[\omega_{2}\right]^{2} \rightarrow \omega_{1}$ establishing $\omega_{2} \nrightarrow\left[\left(\omega_{1}: \omega\right)\right]_{\omega_{1}}^{2}$.

Since Hajnal's proof was never published we sketch his argument. 
Proof of Proposition 2.1. Assume GCH in the ground model. Define a poset $\mathcal{P}=\langle P, \leq\rangle$ as follows. The underlying set $P$ consists of triples $\langle c, \mathcal{A}, \xi\rangle$ where $c:[\operatorname{supp}(c)]^{2} \rightarrow \omega$ for some $\operatorname{supp}(c) \in\left[\omega_{2}\right]^{\omega}, \mathcal{A} \subset[\operatorname{supp}(c)]^{\omega}$ is a countable family and $\xi \in \omega_{1}$.

Put $\langle d, \mathcal{B}, \zeta\rangle \leq\langle c, \mathcal{A}, \xi\rangle$ iff

$(\mathrm{P} 1) c \subset d, \mathcal{A} \subset \mathcal{B}, \xi \leq \zeta$,

(P2) for each $A \in \mathcal{A}$ and for each $\beta \in(\operatorname{supp}(d) \backslash \operatorname{supp}(c)) \cap \min A$,

$$
\xi \subset d^{\prime \prime}[\{\beta\}, A] .
$$

Then $\mathcal{P}$ is a $\sigma$-complete, $\omega_{2}$-c.c. poset and if $\mathcal{G}$ is the generic filter for $\mathcal{P}$ then $g=\bigcup\{c:\langle c, \mathcal{A}, \xi\rangle \in \mathcal{G}\}$ establishes $\omega_{2} \nrightarrow\left[\left(\omega_{1} ; \omega\right)\right]_{\omega_{1}}^{2}$ in $V[\mathcal{G}]$.

Proposition 2.1 validates the following question of Erdös and Hajnal, [6, Problem 6.b]: Assume that a graph $G$ establishes $\omega_{2} \nrightarrow\left(\omega_{1}+\omega\right)_{2}^{2}$. Do all graphs of cardinality $\aleph_{1}$ embed into $G$ ?

To answer this question in the negative we prove a preservation theorem which enables us to apply Shelah's method used to prove [10, Theorem 4.1]: It is consistent that GCH holds and there is colouring $g:\left[\omega_{2}\right]^{2} \rightarrow 2$ establishing $\omega_{2} \nrightarrow\left[\left(\omega_{1}, \omega_{2}\right)\right]_{2}$, but some colouring $h:\left[\omega_{1}\right]^{2} \rightarrow 2$ does not embed into $g$. Shelah actually argued in the following way. He proved two statements:

(a) Assume that $\kappa, \lambda$ and $\tau$ are cardinals of cofinality greater than $\omega$ and $g:[\kappa]^{2} \rightarrow 2$. Then the property

(*) $g$ establishes $\kappa \nrightarrow[(\lambda, \tau)]_{2}$

cannot be destroyed by adding a single Cohen real, i.e. if $V \models(*)$ then $V^{\mathrm{Fn}(\omega, 2)} \models(*)$.

(b) If you add a Cohen real to some model $V$ then in the generic extension there is a colouring $c:\left[\omega_{1}\right]^{2} \rightarrow 2$ which does not embed into any colouring $g$ from $V$.

Hence starting from a model of GCH the generic extension $V^{\mathrm{Fn}(\omega, 2)}$ works because any model of GCH contains a colouring $g$ which establishes $\omega_{2} \nrightarrow$ $\left[\left(\omega_{1}, \omega_{2}\right)\right]_{2}$.

The proof of (a) is based on the observation that if $\operatorname{cf}(\mu)>\omega$ then every "new" subset $A \in V^{\mathrm{Fn}(\omega, 2)}$ of ordinals with cardinality $\mu$ contains an "old" subset $A^{\prime} \in V$ of size $\mu$. This argument does not entail that the property " $g$ establishes $\omega_{2} \nrightarrow\left[\left(\omega_{1}: \omega\right)\right]_{2}$ " cannot be destroyed by adding a single Cohen real because there are countable subsets of ordinals in $V^{\mathrm{Fn}(\omega, 2)}$ which do not contain any infinite set from the ground model.

So, if we want to apply (b) in a similar way, we should prove the following theorem in a different way. 
THEOREM 2.2. If $\mu \leq \omega_{1}$ and $c$ establishes $\omega_{2} \nrightarrow\left[\left(\omega_{1}: \omega\right)\right]_{\mu}^{2}$ then $V^{\mathrm{Fn}(\kappa, 2)}=c$ establishes $\omega_{2} \nrightarrow\left[\left(\omega_{1}: \omega\right)\right]_{\mu}^{2}$.

Proof. The following lemma is straightforward.

Lemma 2.3. Let $\mu \leq \omega_{1}$ and $c:\left[\omega_{2}\right]^{2} \rightarrow \mu$. The following are equivalent:

(1) c establishes $\omega_{2} \nrightarrow\left[\left(\omega_{1} ; \omega\right)\right]_{\mu}^{2}$,

(2) $\left(\forall B \in\left[\omega_{2}\right]^{\omega}\right)(\forall \nu \in \mu)$

$$
\left|\left\{\alpha<\min B: \nu \notin c^{\prime \prime}[\{\alpha\}, B]\right\}\right| \leq \omega,
$$

(3) $\left(\forall \mathcal{B} \in\left[\left[\omega_{2}\right]^{\omega}\right]^{\omega}\right)(\forall \nu \in \mu)$

$$
\left|\left\{\alpha<\min \bigcup \mathcal{B}:(\exists B \in \mathcal{B}) \nu \notin c^{\prime \prime}[\{\alpha\}, B]\right\}\right| \leq \omega .
$$

Suppose on the contrary that the theorem fails to hold. We can assume that we add just $\omega_{1}$ many Cohen reals to $V$, i.e. $\kappa=\omega_{1}$. We can choose $\xi \in \omega_{2}, \nu \in \mu, p \in \operatorname{Fn}\left(\omega_{1}, 2\right)$ and names $\dot{A}$ and $\dot{B}$ such that

$$
p \Vdash \dot{A} \in[\xi]^{\omega_{1}} \wedge \dot{B} \in\left[\omega_{2} \backslash \xi\right]^{\omega} \wedge \nu \notin c^{\prime \prime}[\dot{A}, \dot{B}] .
$$

We can assume that $\dot{B} \in V^{\mathrm{Fn}(\omega, 2)}$ and $\operatorname{dom} p \subset \omega$. For each $q \in \operatorname{Fn}(\omega, 2)$ with $q \leq p$ put

$$
B(q)=\{\zeta:(\exists r \in \operatorname{Fn}(\omega, 2)) r \leq q \wedge r \Vdash \zeta \in \dot{B}\} .
$$

Let $\mathcal{B}=\{B(q): q \in \operatorname{Fn}(\omega, 2), q \leq p\}$ and $A^{\prime}=\left\{\alpha \in \omega_{2}:(\exists r \leq p) r \Vdash\right.$ $\alpha \in \dot{A}\}$. Then $A^{\prime} \in[\xi]^{\omega_{1}}$ and $\mathcal{B} \in\left[\left[\omega_{2} \backslash \xi\right]^{\omega}\right]^{\omega}$. Hence, by Lemma 2.3, there is $\alpha \in A^{\prime}$ such that $\nu \in c^{\prime \prime}[\{\alpha\}, B(q)]$ for each $q \in \operatorname{Fn}(\omega, 2)$ with $q \leq p$. Pick $s \in \operatorname{Fn}\left(\omega_{1}, 2\right)$ with $s \Vdash \alpha \in \dot{A}$. Then $\nu \in c^{\prime \prime}[\{\alpha\}, B(s\lceil\omega)]$, i.e. there are $\beta \in \omega_{2} \backslash \xi$ and $r \in \operatorname{Fn}(\omega, 2)$ such that $r \leq s \uparrow \omega$ and $r \Vdash \beta \in \dot{B}$. Then

$$
s \cup r \Vdash \alpha \in \dot{A} \wedge \beta \in \dot{B} \wedge \nu \notin c^{\prime \prime}[\dot{A}, \dot{B}],
$$

but $c(\alpha, \beta)=\nu$. Contradiction.

THEOREM 2.4. For $2 \leq \mu \leq \omega_{1}$ it is consistent that GCH holds and there is a colouring $f:\left[\omega_{2}\right]^{2} \rightarrow \mu$ establishing $\omega_{2} \nrightarrow\left[\left(\omega_{1}: \omega\right)\right]_{\mu}^{2}$ such that $g \nRightarrow f$ for some colouring $g:\left[\omega_{1}\right]^{2} \rightarrow 2$.

Proof. By Proposition 2.1 we can assume that in the ground model GCH holds and there is a function $f:\left[\omega_{2}\right]^{2} \rightarrow \omega_{1}$ establishing $\omega_{2} \nrightarrow\left[\left(\omega_{1}: \omega\right)\right]_{\omega_{1}}$.

If $\mu \leq \omega_{1}$ and $\pi_{\mu}: \omega_{1} \rightarrow \mu$ is onto then the function $f_{\mu}=\pi_{\mu} \circ f$ establishes $\omega_{2} \nrightarrow\left[\left(\omega_{1}: \omega\right)\right]_{\mu}$. Then, by [10, Theorem 4.1], in $V^{\mathrm{Fn}(\omega, 2)}$ there is a function $d:\left[\omega_{1}\right]^{2} \rightarrow 2$ such that $d \nRightarrow f_{\mu}$.

Since

$$
V^{\mathrm{Fn}(\omega, 2)} \models f_{\mu} \text { establishes } \omega_{2} \nrightarrow\left[\left(\omega_{1}: \omega\right)\right]_{\mu}
$$

by Theorem 2.2, we are done.

As observed by Hajnal, the construction of Theorem 2.4 above left open the following question which he raised in [9, Problem 4.1]: 
Problem. Assume GCH holds and a colouring $c:\left[\omega_{2}\right]^{2} \rightarrow \omega_{1}$ establishes $\omega_{2} \nrightarrow\left[\left(\omega_{1}: \omega\right)\right]_{\omega_{1}}$. Does there exist a c-rainbow set of size $\omega_{1}$ ?

Before answering this question let us recall some positive results of Hajnal. In [9], he proved that

THEOREM.

(1) If $f:\left[\omega_{1}\right]^{2} \rightarrow \omega_{1}$ establishes $\omega_{1} \nrightarrow\left[\left(\omega_{1} ; \omega_{1}\right)\right]_{\omega_{1}}$ then $d \Rightarrow f$ for each $d:[\omega]^{2} \rightarrow \omega_{1}$.

(2) If $f:\left[\omega_{1}\right]^{2} \rightarrow \omega$ establishes $\omega_{1} \nrightarrow\left[\left(\omega_{1}, \omega_{1}\right)\right]_{\omega}$ then there exists an infinite $f$-rainbow set.

When we colour the pairs of $\omega_{1}$ we cannot expect uncountable rainbow sets because of the following fact.

Proposition 2.5. If $\mathrm{CH}$ holds then there is a function $f:\left[\omega_{1}\right]^{2} \rightarrow \omega_{1}$ such that

(1) $f$ establishes $\omega_{1} \nrightarrow\left[\left(\omega, \omega_{1}\right)\right]_{\omega_{1}}$,

(2) there is no uncountable $f$-rainbow.

Proof. Enumerate $\left[\omega_{1}\right]^{\omega}$ as $\left\{A_{\alpha}: \omega \leq \alpha<\omega_{1}\right\}$ such that $A_{\alpha} \subset \alpha$. By induction on $\alpha, \omega \leq \alpha<\omega_{1}$, define $f(\xi, \alpha)$ for $\xi<\alpha$ such that

(i) $\beta \subset\left\{f(\xi, \beta): \xi \in A_{\alpha}\right\}$ for $\alpha<\beta$,

(ii) $A_{\alpha} \cup\{\beta\}$ is not an $f$-rainbow for $\alpha<\beta$.

Let $A \in\left[\omega_{1}\right]^{\omega}, B \in\left[\omega_{1}\right]^{\omega_{1}}$ and $\sigma \in \omega_{1}$. Then $A=A_{\alpha}$ for some $\alpha<\omega_{1}$. Let $\beta \in B \backslash \max (\alpha, \sigma)$. Then $\sigma \in f^{\prime \prime}\left[A_{\alpha},\{\beta\}\right] \subset f^{\prime \prime}[A, B]$ by (i). So (1) holds.

If $A \in\left[\omega_{1}\right]^{\omega_{1}}$ then choose $\alpha<\omega_{1}$ such that $A_{\alpha} \in[A]^{\omega}$ and then pick $\beta \in A \backslash \alpha$. Thus $A_{\alpha} \cup\{\beta\}$ is not an $f$-rainbow by (ii), so we have (2).

Next we answer [9, Problem 4.1] in the negative.

THEOREM 2.6. It is consistent that GCH holds and there is a function $g:\left[\omega_{2}\right]^{2} \rightarrow \omega_{1}$ such that

(1) $g$ establishes $\omega_{2} \nrightarrow\left[\left(\omega_{1}: \omega\right)\right]_{\omega_{1}}^{2}$,

(2) there is no uncountable g-rainbow.

Proof. Assume GCH in the ground model.

The naive approach is to try to modify the order of the poset $P$ from the proof of Proposition 2.1 by adding a condition (P3) to the definition of the order:

(P3) for each $A \in \mathcal{A}$ and for each $\beta \in \operatorname{supp}(d) \backslash \operatorname{supp}(c)$ the set $A \cup\{\beta\}$ is not a $d$-rainbow.

Unfortunately this approach does not work because the modified poset does not satisfy $\omega_{2}$-c.c. 
Indeed, we can construct an antichain of size $\omega_{2}$ as follows. Let $f:[\omega]^{2}$ $\rightarrow \omega$ be a bijection. Fix a partition $\left\{A_{n}: n<\omega\right\}$ of $\omega \backslash\{0,1\}$ into infinite pieces such that $n \notin f^{\prime \prime}\left[A_{n}\right]^{2} \cup f^{\prime \prime}\left[A_{n},\{0,1\}\right]$ for $n<\omega$. Let $\left\{\alpha_{\nu}, \beta_{\nu}\right.$ : $\left.\nu<\omega_{2}\right\} \subset \omega_{2} \backslash \omega$ be pairwise different ordinals. For each $\nu<\omega_{2}$ define a condition $p_{\nu}=\left\langle c_{\nu}, \mathcal{A}_{\nu}, 0\right\rangle$ as follows:

- $\operatorname{supp}\left(c_{\nu}\right)=(\omega \backslash 2) \cup\left\{\alpha_{\nu}, \beta_{\nu}\right\}$,

- $c_{\nu} \uparrow[\omega \backslash 2]^{2}=f \uparrow[\omega \backslash 2]^{2}$,

- $c_{\nu}\left(k, \alpha_{\nu}\right)=f(k, 0)$ for $k \in \omega \backslash 2$,

- $c_{\nu}\left(k, \beta_{\nu}\right)=f(k, 1)$ for $k \in \omega \backslash 2$,

- $f\left(\alpha_{\nu}, \beta_{\nu}\right)=0$,

- $\mathcal{A}_{\nu}=\left\{A_{n} \cup\left\{\alpha_{\nu}\right\}: n<\omega\right\}$.

Suppose on the contrary that $q$ is a common extension of $p_{\nu}$ and $p_{\mu}$ for some $\nu \neq \mu<\omega_{2}$. Let $n=q\left(\alpha_{\nu}, \beta_{\mu}\right)$. Then $A_{n} \cup\left\{\alpha_{\nu}\right\} \cup\left\{\beta_{\mu}\right\}$ is a $q$-rainbow, which contradicts (P3) because $A_{n} \cup\left\{\alpha_{\nu}\right\} \in \mathcal{A}_{\nu}$.

So we will argue in a different way. Define a poset $P$ as follows. The underlying set $P$ consists of quadruples $\langle c, \mathcal{A}, \xi, \mathcal{D}\rangle$ where

(i) $c:[\operatorname{supp}(c)]^{2} \rightarrow \omega$ for some $\operatorname{supp}(c) \in\left[\omega_{2}\right]^{\omega}$,

(ii) $\mathcal{A} \subset[\operatorname{supp}(c)]^{\omega}$ is a countable family,

(iii) $\omega \leq \xi<\omega_{1}$,

(iv) $\mathcal{D} \subset[\operatorname{supp}(c)]^{\omega} \times \omega_{1}$ is a countable family,

(v) $(\forall\langle D, \sigma\rangle \in \mathcal{D})(\forall \gamma \in \operatorname{supp}(c))|\{\delta \in D: c(\gamma, \delta)<\sigma\}|=\omega$.

Put $\langle d, \mathcal{B}, \zeta, \mathcal{E}\rangle \preceq\langle c, \mathcal{A}, \xi, \mathcal{D}\rangle$ iff

(a) $c \subset d, \mathcal{A} \subset \mathcal{B}, \xi \leq \zeta, \mathcal{D} \subset \mathcal{E}$,

(b) for all $A \in \mathcal{A}$ and $\beta \in(\operatorname{supp}(d) \backslash \operatorname{supp}(c)) \cap \min A$,

$$
\xi \subset d^{\prime \prime}[\{\beta\}, A] \text {. }
$$

Clearly $\preceq$ is a partial order on $P$ and $\mathcal{P}=\langle P, \preceq\rangle$ is $\sigma$-complete.

Lemma 2.7. $\mathcal{P}$ is $\omega_{2}$-c.c.

Proof. For subsets $A$ and $B$ or ordinals we write $A<B$ iff $\sup (A)<$ $\min (B)$. We say that two conditions, $p=\langle c, \mathcal{A}, \xi, \mathcal{D}\rangle$ and $p^{\prime}=\left\langle c^{\prime}, \mathcal{A}^{\prime}, \xi^{\prime}, \mathcal{D}^{\prime}\right\rangle$, are twins iff there is an order preserving bijection $\varphi: \operatorname{supp}(c) \rightarrow \operatorname{supp}\left(c^{\prime}\right)$ such that

(1) $K=\operatorname{supp}(c) \cap \operatorname{supp}\left(c^{\prime}\right)$ is an initial segment of both $\operatorname{supp}(c)$ and $\operatorname{supp}\left(c^{\prime}\right)$

(2) $K<\operatorname{supp}(c) \backslash K<\operatorname{supp}\left(c^{\prime}\right) \backslash K$,

(3) $c(\xi, \eta)=c^{\prime}(\varphi(\xi), \varphi(\eta))$ for each $\{\xi, \eta\} \in[\operatorname{supp}(c)]^{2}$,

(4) $\mathcal{A}^{\prime}=\left\{\varphi^{\prime \prime} A: A \in \mathcal{A}\right\}$,

(5) $\xi=\xi^{\prime}$,

(6) $\mathcal{D}^{\prime}=\left\{\left\langle\varphi^{\prime \prime} D, \sigma\right\rangle:\langle D, \sigma\rangle \in \mathcal{D}\right\}$. 
Since $\mathrm{CH}$ holds, standard counting and $\Delta$-system arguments show that any subset of $P$ of cardinality $\omega_{2}$ contains two elements which are twins. So it is enough to show that if $p$ and $p^{\prime}$ are twins then they have a common extension $q=\langle d, \mathcal{B}, \varrho, \mathcal{E}\rangle$. Let $\mathcal{B}=\mathcal{A} \cup \mathcal{A}^{\prime}, \varrho=\xi=\xi^{\prime}$ and $\mathcal{E}=\mathcal{D} \cup \mathcal{D}^{\prime}$.

We should define $d(\nu, \mu)$ for $\nu \in \operatorname{supp}(c) \backslash K$ and $\mu \in \operatorname{supp}\left(c^{\prime}\right) \backslash K$.

We enumerate all "tasks" as follows: Let

$$
\begin{gathered}
\mathcal{T}_{0}=\left\{\left\langle\beta, A^{\prime}, \zeta\right\rangle: \beta \in \operatorname{supp}(c) \backslash K, A^{\prime} \in \mathcal{A}^{\prime}, A^{\prime} \subset \operatorname{supp}\left(c^{\prime}\right) \backslash K, \zeta<\xi^{\prime}\right\}, \\
\mathcal{T}_{1}=\left\{\left\langle\gamma,\left\langle D^{\prime}, \sigma^{\prime}\right\rangle, n\right\rangle: \gamma \in \operatorname{supp}(c) \backslash K,\right. \\
\left.\qquad\left\langle D^{\prime}, \sigma^{\prime}\right\rangle \in \mathcal{D}^{\prime} \backslash \mathcal{D},\left|D^{\prime} \backslash K\right|=\omega, n<\omega\right\}, \\
\mathcal{T}_{2}=\left\{\left\langle\gamma^{\prime},\langle D, \sigma\rangle, n\right\rangle: \gamma^{\prime} \in \operatorname{supp}\left(c^{\prime}\right) \backslash K,\right. \\
\left.\quad\langle D, \sigma\rangle \in \mathcal{D} \backslash \mathcal{D}^{\prime},|D \backslash K|=\omega, n<\omega\right\} .
\end{gathered}
$$

Since $\mathcal{T}=\mathcal{T}_{0} \cup \mathcal{T}_{1} \cup \mathcal{T}_{2}$ is countable we can pick pairwise distinct ordinals $\left\{\eta_{x}: x \in \mathcal{T}\right\}$ such that

- if $x=\left\langle\beta, A^{\prime}, \zeta\right\rangle \in \mathcal{T}_{0}$ then $\eta_{x} \in A^{\prime}$,

- if $x=\left\langle\gamma,\left\langle D^{\prime}, \sigma^{\prime}\right\rangle, n\right\rangle \in \mathcal{T}_{1}$ then $\eta_{x} \in D^{\prime} \backslash K$,

- if $x=\left\langle\gamma^{\prime},\langle D, \sigma\rangle, n\right\rangle \in \mathcal{T}_{2}$ then $\eta_{x} \in D \backslash K$.

Choose a function $d:\left[\operatorname{supp}(c) \cup \operatorname{supp}\left(c^{\prime}\right)\right]^{2} \rightarrow \omega_{1}$ such that

- $d \supset c \cup c^{\prime}$,

- $d\left(\beta, \eta_{x}\right)=\zeta$ for $x=\left\langle\beta, A^{\prime}, \zeta\right\rangle \in \mathcal{T}_{0}$,

- $d\left(\gamma, \eta_{x}\right)=0$ for $x=\left\langle\gamma,\left\langle D^{\prime}, \sigma^{\prime}\right\rangle, n\right\rangle \in \mathcal{T}_{1}$,

- $d\left(\gamma^{\prime}, \eta_{x}\right)=0$ for $x=\left\langle\gamma^{\prime},\langle D, \sigma\rangle, n\right\rangle \in \mathcal{T}_{2}$.

Let $q=\langle d, \mathcal{B}, \varrho, \mathcal{E}\rangle$. To show $q \in P$ we need only check condition (v). So let $\langle D, \sigma\rangle \in \mathcal{E}$ and $\gamma \in \operatorname{supp}(d)$. Assume that $\langle D, \sigma\rangle \in \mathcal{D}$. (The case $\langle D, \sigma\rangle \in \mathcal{D}^{\prime}$ is similar.)

If $\gamma \in \operatorname{supp}(c)$ then $d \uparrow[\{\gamma\}, D]=c \uparrow[\{\gamma\}, D]$ so we are done. So we can assume that $\gamma \in \operatorname{supp}\left(c^{\prime}\right) \backslash K$.

If $D \backslash K$ is finite then the set

$$
E=\left\{\delta \in D \cap K: c\left(\delta, \varphi^{-1}(\gamma)\right)<\sigma\right\}
$$

is infinite because $p \in P$ satisfies (v) and for each $\delta \in E$ we have $d(\delta, \gamma)=$ $c^{\prime}(\delta, \gamma)=c^{\prime}(\varphi(\delta), \gamma)=c\left(\delta, \varphi^{-1}(\gamma)\right)<\sigma$. So we can assume that $D \backslash K$ is infinite. In this case $x_{n}=\langle\gamma,\langle D, \sigma\rangle, n\rangle \in \mathcal{T}_{2}$ for $n \in \omega$, so $d\left(\gamma, \eta_{x_{n}}\right)=0<\sigma$ and $\left\{\eta_{x_{n}}: n \in \omega\right\} \in[D]^{\omega}$. So $q \in P$.

It is straightforward that $q \preceq p$ because no instances of (b) should be checked.

Finally, we verify $q \preceq p^{\prime}$. Since condition (a) is clear, assume that $A^{\prime} \in \mathcal{A}^{\prime}$ and $\beta \in \operatorname{supp}(c) \backslash K$ with $\beta<\min A^{\prime}$. Since $\sup K<\beta$ we have $A^{\prime} \subset$ $\operatorname{supp}\left(c^{\prime}\right) \backslash K$. Hence for each $\zeta<\xi$ we have $x=\left\langle\beta, A^{\prime}, \zeta\right\rangle \in \mathcal{T}_{0}$ so $d\left(\beta, \eta_{x}\right)=\zeta$. Thus $\xi \subset d^{\prime \prime}\left[\{\beta\}, A^{\prime}\right]$.

This completes the proof of the lemma. 
Let $\mathcal{G}$ be the generic filter for $\mathcal{P}$ and put $g=\bigcup\{c:\langle c, \mathcal{A}, \xi\rangle \in \mathcal{G}\}$.

Claim. g establishes $\omega_{2} \nrightarrow\left[\left(\omega_{1} ; \omega\right)\right]_{\omega_{1}}^{2}$ in $V[\mathcal{G}]$.

Indeed, let $p=\langle c, \mathcal{A}, \xi, \mathcal{D}\rangle \in P$. If $A \in[\operatorname{supp}(c)]^{\omega}$ and $\eta \in \omega_{1}$ then $p^{\prime}=\langle c, \mathcal{A} \cup\{A\}, \max (\xi, \eta), \mathcal{D}\rangle \preceq p$ and for each $\beta \in \min A \backslash \operatorname{supp}(c)$,

$$
p^{\prime} \Vdash \eta \subset g^{\prime \prime}[\{\beta\}, A] .
$$

Claim. There is no uncountable g-rainbow set in $V[\mathcal{G}]$.

Indeed, assume that $p_{0} \Vdash \dot{X} \in\left[\omega_{2}\right]^{\omega_{1}}$. Since $\mathcal{P}$ is $\sigma$-complete there are $p \preceq p_{0}, p=\langle c, \mathcal{A}, \xi, \mathcal{D}\rangle$, and $D \in[\operatorname{supp}(c)]^{\omega}$ such that $p \Vdash D \subset \dot{X}$. Let $p^{\prime}=\langle c, \mathcal{A}, \xi, \mathcal{D} \cup\{\langle D,(\sup \operatorname{ran}(c))+1\rangle\}$.$\rangle . Then p^{\prime} \in P$ and $p^{\prime} \preceq p$. Moreover, $p^{\prime} \Vdash \dot{X}$ is not a $g$-rainbow.

Indeed, work in $V[\mathcal{G}]$, where $p^{\prime} \in \mathcal{G}$. Write $X=\left\{\xi_{\nu}: \nu \in \omega_{1}\right\}$. Then for each $\nu<\omega$ there are $\gamma_{\nu}<\sup \operatorname{ran}(c)+1$ and $\delta_{\nu} \in D$ with $g\left(\delta_{\nu}, \xi_{\nu}\right)=\gamma_{\nu}$. Then there are $\nu<\mu<\omega_{1}$ with $\gamma_{\nu}=\gamma_{\mu}$. Hence $g\left(\delta_{\nu}, \xi_{\nu}\right)=\gamma_{\nu}=\gamma_{\mu}=g\left(\delta_{\mu}, \xi_{\mu}\right)$ and $\xi_{\nu} \neq \xi_{\mu}$, i.e. $X$ is not a $g$-rainbow.

So, by the claims above, $g$ satisfies the requirements of the theorem.

In his Ph.D. thesis [3], Baumgartner proved that if $\mathrm{CH}$ holds and $P=$ $\operatorname{Fn}\left([\kappa]^{2}, \omega_{1} ; \omega_{1}\right)$ for some cardinal $\kappa \geq \omega_{2}$, and $\mathcal{G}$ is the generic filter above $P$, then the function $g=\bigcup \mathcal{G}$ establishes $\kappa \nrightarrow\left[\left(\omega_{1}, \omega_{2}\right)\right]_{\omega_{1}}^{2}$. The original proof is not easily available, but a "stripped-down" version of the next proof gives Baumgartner's result (just remove the "side conditions").

THEOREM 2.8. If $C H$ holds and $\kappa \geq \omega_{2}$ is a cardinal then there is a $\sigma$ complete, $\omega_{2}$-c.c. poset $P$ such that in $V^{P}$ there is a function $g:[\kappa]^{2} \rightarrow \omega_{1}$ such that

(1) g establishes $\kappa \nrightarrow\left[\left(\omega_{1}, \omega_{2}\right)\right]_{\omega_{1}}^{2}$.

(2) there is no uncountable g-rainbow subset of $\kappa$.

Proof. Define poset $\mathcal{P}=\langle P, \preceq\rangle$ as follows. The underlying set $P$ consists of pairs $\langle c, \mathcal{D}\rangle$ where

(i) $c:[\operatorname{supp}(c)]^{2} \rightarrow \omega$ for some $\operatorname{supp}(c) \in[\kappa]^{\omega}$,

(ii) $\mathcal{D} \subset[\operatorname{supp}(c)]^{\omega} \times \omega_{1}$ is a countable family,

(iii) $(\forall\langle D, \sigma\rangle \in \mathcal{D})(\forall \gamma \in \operatorname{supp}(c))|\{\delta \in D: c(\gamma, \delta)<\sigma\}|=\omega$.

Put $\langle d, \mathcal{E}\rangle \preceq\langle c, \mathcal{D}\rangle$ iff $c \subset d$ and $\mathcal{D} \subset \mathcal{E}$. Then $\preceq$ is a partial order, and $\mathcal{P}$ is $\sigma$-complete.

We say that two conditions, $p=\langle c, \mathcal{D}\rangle$ and $p^{\prime}=\left\langle c^{\prime}, \mathcal{D}^{\prime}\right\rangle$, are twins iff there is an order preserving bijection $\varphi: \operatorname{supp}(c) \rightarrow \operatorname{supp}\left(c^{\prime}\right)$ such that

- $\varphi(\xi)=\xi$ for $\xi \in \operatorname{supp}(c) \cap \operatorname{supp}\left(c^{\prime}\right)$,

- $c(\xi, \eta)=c^{\prime}(\varphi(\xi), \varphi(\eta))$ for each $\{\xi, \eta\} \in[\operatorname{supp}(c)]^{2}$,

- $\mathcal{D}^{\prime}=\left\{\left\langle\varphi^{\prime \prime} D, \sigma\right\rangle:\langle D, \sigma\rangle \in \mathcal{D}\right\}$. 
Lemma 2.9. Assume that $p=\langle c, \mathcal{D}\rangle, p^{\prime}=\left\langle c^{\prime}, \mathcal{D}^{\prime}\right\rangle$ are twins. Let $q \preceq p$, $q=\langle d, \mathcal{E}\rangle$, be such that $\operatorname{supp}(d) \cap \operatorname{supp}\left(c^{\prime}\right)=\operatorname{supp}(c) \cap \operatorname{supp}\left(c^{\prime}\right)$. Let $A \in$ $\left[\operatorname{supp}(d) \backslash \operatorname{supp}\left(c^{\prime}\right)\right]^{\omega}, \xi \in \operatorname{supp}\left(c^{\prime}\right) \backslash \operatorname{supp}(c)$ and $\varrho<\omega_{1}$. Then there is a common extension $r=\left\langle c_{r}, \mathcal{D}_{r}\right\rangle$ of $q$ and $p^{\prime}$ such that $\varrho \subset c_{r}^{\prime \prime}[\{\xi\}, A]$.

Proof. Write $K=\operatorname{supp}(c) \cap \operatorname{supp}\left(c^{\prime}\right)$ and fix a function $\varphi$ witnessing that $p$ and $p^{\prime}$ are twins. Let

$$
\begin{aligned}
& \mathcal{T}_{0}=\varrho, \\
& \mathcal{T}_{1}=\left\{\left\langle\gamma,\left\langle D^{\prime}, \sigma^{\prime}\right\rangle, n\right\rangle: \gamma \in \operatorname{supp}(d) \backslash K,\left\langle D^{\prime}, \sigma^{\prime}\right\rangle \in \mathcal{D}^{\prime},\left|D^{\prime} \backslash K\right|=\omega, n \in \omega\right\}, \\
& \mathcal{T}_{2}=\left\{\left\langle\gamma^{\prime},\langle E, \sigma\rangle, n\right\rangle: \gamma^{\prime} \in \operatorname{supp} c^{\prime} \backslash K,\langle E, \sigma\rangle \in \mathcal{E},|E \backslash K|=\omega, n \in \omega\right\} .
\end{aligned}
$$

Since $\mathcal{T}=\mathcal{T}_{0} \cup \mathcal{T}_{1} \cup \mathcal{T}_{2}$ is countable we can pick pairwise distinct ordinals $\left\{\eta_{x}: x \in \mathcal{T}\right\}$ such that

(a) if $x=\chi \in \varrho$ then $\eta_{x} \in A$,

(b) if $x=\langle\gamma,\langle D, \sigma\rangle, n\rangle \in \mathcal{T}_{1} \cup \mathcal{T}_{2}$ then $\eta_{x} \in D \backslash K$.

Let $c_{r} \supset d \cup c_{\nu}$ be such that

- $c_{r}\left(\eta_{x}, \xi\right)=\chi$ if $x=\chi \in \mathcal{T}_{0}$,

- $c_{r}\left(\eta_{x}, \gamma\right)=0$ if $\langle\gamma,\langle D, \sigma\rangle, n\rangle \in \mathcal{T}_{0} \cup \mathcal{T}_{1}$.

To prove $r=\left\langle c_{r}, \mathcal{D}^{\prime} \cup \mathcal{E}\right\rangle \in P$ it is enough to check condition (iii).

Assume first that $\langle D, \sigma\rangle \in \mathcal{D}^{\prime}$. If $\gamma \in \operatorname{supp}\left(c^{\prime}\right)$ then $c_{r}\lceil[\{\gamma\}, D]=$ $c^{\prime} \uparrow[\{\gamma\}, D]$ so we are done. So we can assume that $\gamma \in \operatorname{supp}(d) \backslash K$.

If $D \backslash K$ is finite then $\left\langle\varphi^{-1} D, \sigma\right\rangle \in \mathcal{D} \subset \mathcal{E}$ and $D \cap K=\varphi^{-1} D \cap K$, so the set

$$
H=\{\delta \in D \cap K: d(\delta, \gamma)<\sigma\}
$$

is infinite because $q \in P$ satisfies (iii), and $H \subset\left\{\delta \in D: c_{r}(\delta, \gamma)<\sigma\right\}$.

So we can assume that $D \backslash K$ is infinite. In this case $x_{n}=\langle\gamma,\langle D, \sigma\rangle, n\rangle \in$ $\mathcal{T}_{1}$ for $n \in \omega$, so $c_{r}\left(\gamma, \eta_{x_{n}}\right)=0<\sigma$ and $\left\{\eta_{x_{n}}: n \in \omega\right\} \in[D]^{\omega}$.

Assume now that $\langle D, \sigma\rangle \in \mathcal{E}$. If $\gamma \in \operatorname{supp}(d)$ then $c_{r} \uparrow[\{\gamma\}, D]=d \uparrow[\{\gamma\}, D]$ so we are done. So we can assume that $\gamma \in \operatorname{supp}\left(c^{\prime}\right) \backslash K$.

If $D \backslash K$ is finite then $\gamma^{\prime}=\varphi^{-1}(\gamma) \in \operatorname{supp}(c) \subset \operatorname{supp}(d)$ and $q \in P$ imply that the set

$$
H=\left\{\varepsilon \in D \cap K: d\left(\varepsilon, \gamma^{\prime}\right)<\sigma\right\}
$$

is infinite. But for each $\varepsilon \in H$ we have $c_{r}(\varepsilon, \gamma)=c^{\prime}(\varepsilon, \gamma)=c\left(\varepsilon, \gamma^{\prime}\right)=d\left(\varepsilon, \gamma^{\prime}\right)$. So we can assume that $D \backslash K$ is infinite. In this case $x_{n}=\langle\gamma,\langle D, \sigma\rangle, n\rangle \in \mathcal{T}_{2}$ for $n \in \omega$, so $c_{r}\left(\gamma, \eta_{x_{n}}\right)=0<\sigma$ and $\left\{\eta_{x_{n}}: n \in \omega\right\} \in[D]^{\omega}$. So $r \in P$ and clearly $r \preceq q, p^{\prime}$.

Finally, for each $\zeta<\varrho$ we have $\eta_{\zeta} \in A$ and $c_{r}\left(\xi, \eta_{\zeta}\right)=\zeta$. So $\varrho \subset$ $c_{r}^{\prime \prime}[\{\xi\}, A]$.

LEMma 2.10. $\mathcal{P}$ is $\omega_{2}$-c.c. 
Proof. Since any family of conditions of size $\omega_{2}$ contains two conditions $p$ and $p^{\prime}$ which are twins we can apply the previous lemma to show that $p$ and $p^{\prime}$ are compatible in $P$.

Let $\mathcal{G}$ be the generic filter for $\mathcal{P}$ and put $g=\bigcup\{c:\langle c, \mathcal{A}, \xi\rangle \in \mathcal{G}\}$

LEMMA 2.11. g establishes $\kappa \nrightarrow\left[\left(\omega_{1}, \omega_{2}\right)\right]_{\omega_{1}}$ in $V[\mathcal{G}]$.

Proof. Assume that $p \Vdash \dot{X}=\left\{\dot{\xi}_{\nu}: \nu<\omega_{2}\right\} \in[\kappa]^{\omega_{2}}, \dot{Y} \in[\kappa]^{\omega_{1}}$.

For each $\varrho<\omega_{1}$ we will construct a condition $r \preceq p$ such that $r \Vdash \varrho \subset$ $g^{\prime \prime}[\dot{X}, \dot{Y}]$. Write $p=\langle c, \mathcal{D}\rangle$. For each $\nu<\omega_{2}$ pick $p_{\nu}=\left\langle c_{\nu}, \mathcal{D}_{\nu}\right\rangle \preceq p$ such that $p_{\nu} \Vdash \dot{\xi}_{\nu}=\xi_{\nu}$ for some $\xi_{\nu} \in \operatorname{supp}\left(c_{\nu}\right)$. Since CH holds there is $I \in\left[\omega_{2}\right]^{\omega_{2}}$ such that

- $\left\{\operatorname{supp}\left(c_{\nu}\right): \nu \in I\right\}$ forms a $\Delta$-system with kernel $K$,

- for each $\{\nu, \mu\} \in[I]^{2}$ the conditions $p_{\nu}$ and $p_{\mu}$ are twins.

Since $P$ satisfies $\omega_{2}$-c.c. we can assume that $\xi_{\nu} \in \operatorname{supp}\left(c_{\nu}\right) \backslash K$ for $\nu \in I$.

Fix $\mu \in I$. Pick a condition $q \preceq p_{\mu}, q=\langle d, \mathcal{E}\rangle$, such that $q \Vdash Z \subset \dot{Y}$ for some $Z \in[\operatorname{supp}(d) \cap(\kappa \backslash K)]^{\omega}$. Choose $\nu \in I$ such that $\operatorname{supp}\left(c_{\nu}\right) \cap \operatorname{supp}(d)$ $=K$.

By Lemma 2.9 there is a condition $r=\left\langle c_{r}, \mathcal{D}_{\nu} \cup \mathcal{E}\right\rangle \in P$ such that $r \preceq q, p_{\nu}$ and $\varrho \subset c_{r}^{\prime \prime}\left[\left\{\xi_{\mu}\right\}, Z\right]$. Then $r \Vdash \varrho \subset c_{r}^{\prime \prime}\left[\left\{\xi_{\nu}\right\}, Z\right] \subset g^{\prime \prime}[\dot{X}, \dot{Y}]$.

Lemma 2.12. There is no uncountable g-rainbow set in $V[\mathcal{G}]$.

Proof. Indeed, assume that $p_{0} \Vdash \dot{X} \in\left[\omega_{2}\right]^{\omega_{1}}$. Since $\mathcal{P}$ is $\sigma$-complete there are $p \preceq p_{0}, p=\langle c, \mathcal{D}\rangle$, and $D \in[\operatorname{supp}(c)]^{\omega}$ such that $p \Vdash D \subset \dot{X}$. Let $p^{\prime}=\langle c, \mathcal{D} \cup\{\langle D,(\sup \operatorname{ran}(c))+1\rangle\}$.$\rangle . Then p^{\prime} \in P$ and $p^{\prime} \preceq p$. Moreover

$$
p^{\prime} \Vdash \dot{X} \text { is not a } g \text {-rainbow. }
$$

Indeed, work in $V[\mathcal{G}]$, where $p^{\prime} \in \mathcal{G}$. Write $X=\left\{\xi_{\nu}: \nu \in \omega_{1}\right\}$. Then for each $\nu<\omega$ there are $\gamma_{\nu}<\sup \operatorname{ran}(c)+1$ and $\delta_{\nu} \in D$ with $g\left(\delta_{\nu}, \xi_{\nu}\right)=\gamma_{\nu}$. Then there are $\nu<\mu<\omega_{1}$ with $\gamma_{\nu}=\gamma_{\mu}$. Thus $g\left(\delta_{\nu}, \xi_{\nu}\right)=\gamma_{\nu}=\gamma_{\mu}=g\left(\delta_{\mu}, \xi_{\mu}\right)$ and $\xi_{\nu} \neq \xi_{\mu}$, i.e. $X$ is not a $g$-rainbow.

So, by the lemmas above, $g$ satisfies the requirements of the theorem.

\section{3. $k$-bounded colourings}

Definition 3.1. Let $X \in\left[\omega_{1}\right]^{\omega_{1}}, f:[X]^{2} \rightarrow \omega_{1}, k \in \omega$.

(a) $f$ is $k$-bounded iff $\left|f^{-1}\{\gamma\}\right| \leq k$ for each $\gamma \in \operatorname{ran}(f)$.

(b) Put

$$
\mathcal{D}^{(k)}(X)=\left\{D \in\left[[X]^{k}\right]^{<\omega}: d \cap d^{\prime}=\emptyset \text { for each }\left\{d, d^{\prime}\right\} \in[D]^{2}\right\} .
$$

(c) For $D \in \mathcal{D}^{(k)}(X)$ let

$$
\operatorname{Hom}(D, f)=\left\{\alpha:(\forall d \in D)\left(\forall \delta, \delta^{\prime} \in d\right) f(\delta, \alpha)=f\left(\delta^{\prime}, \alpha\right)\right\} .
$$


(d) Given any cardinal $\mu$ let

$\mathbb{D}_{\mu}^{(k)}(X)=\left\{\left\langle D_{i}: i<\mu\right\rangle \subset \mathcal{D}^{(k)}(X):\left(\bigcup D_{i}\right) \cap\left(\bigcup D_{j}\right)=\emptyset\right.$ for $\left.i<j<\mu\right\}$.

(e) $f$ is an $A R^{(k)}$-function iff

- $f$ is $k$-bounded,

- for each $\left\langle D_{i}: i<\omega\right\rangle \in \mathbb{D}_{\omega}^{(k)}(X)$ there is $\gamma<\omega_{1}$ such that

$$
X \backslash \gamma \subset \bigcup\left\{\operatorname{Hom}\left(D_{i}, f\right): i<\omega\right\} \text {. }
$$

OBSERVATion 3.2. An $A R^{(k)}$-function $f:\left[\omega_{1}\right]^{2} \rightarrow \omega_{1}$ establishes the negative partition relation $\omega_{1} \nrightarrow^{*}\left[\left(\omega ; \omega_{1}\right)\right]_{k \text {-bdd }}$.

Proof. Assume that $A \in\left[\omega_{1}\right]^{\omega}$ and $B \in\left[\omega_{1}\right]^{\omega_{1}}$. Pick pairwise disjoint sets $\left\{d_{i}: i<\omega\right\} \subset[A]^{k}$. Write $D_{i}=\left\{d_{i}\right\}$ and $\vec{D}=\left\langle D_{i}: i<\omega\right\rangle$. Since $\vec{D} \in \mathbb{D}_{\omega}^{(k)}\left(\omega_{1}\right)$ and $f$ is an $\operatorname{AR}^{(k)}$-function, there is $\beta \in B$ such that $\beta \in$ $\operatorname{Hom}\left(D_{i}, f\right)$ for some $i<\omega$, which means that $\left|f^{\prime \prime}\left[d_{i},\{\beta\}\right]\right|=1$. Since $d_{i} \in$ $[A]^{k}$ we are done.

LEMMA 3.3. If $C H$ holds then for each $k \in \omega$ there is an $A R^{(k)}$-function $f:\left[\omega_{1}\right]^{2} \rightarrow \omega_{1}$.

Proof. The construction is standard. Let $\left\{C_{\alpha}: \omega \leq \alpha<\omega_{1}\right\} \subset\left[\omega_{1}\right]^{\omega}$ be disjoint sets. Fix an enumeration $\left\langle\vec{D}_{\alpha}: \omega \leq \alpha<\omega_{1}\right\rangle$ of $\mathbb{D}_{\omega}^{(k)}\left(\omega_{1}\right)$ such that $\bigcup \vec{D}_{\alpha} \subset \alpha$.

Let $\alpha<\omega_{1}$ be fixed. For each $\xi<\alpha$ pick $i_{\xi} \in \omega$ such that the sets $\left\{\bigcup \vec{D}_{\xi}\left(i_{\xi}\right): \xi<\alpha\right\}$ are pairwise disjoint. Choose a function $g_{\alpha}: \alpha \rightarrow C_{\alpha}$ such that

$(*) \quad g_{\alpha}(\delta)=g_{\alpha}\left(\delta^{\prime}\right) \quad$ iff $\quad\left\{\delta, \delta^{\prime}\right\} \in[d]^{2}$ for some $\xi<\alpha$ and $d \in \vec{D}_{\xi}\left(i_{\xi}\right)$.

For $\delta<\alpha$ let $f(\delta, \alpha)=g_{\alpha}(\delta)$.

THEOREM 3.4. If $G C H$ holds and $f:\left[\omega_{1}\right]^{2} \rightarrow \omega_{1}$ is an $A R^{(k)}$-function then there is a c.c.c. poset $P$ such that

$$
V^{P} \models f \text { c.c.c.-indestructibly establishes } \omega_{1} \nrightarrow^{*}\left[\left(\omega_{1} ; \omega_{1}\right)\right]_{k \text {-bdd }} \text {. }
$$

We say that $\omega_{1} \nrightarrow^{*}\left[\left(\omega, \omega_{1}\right)\right]_{\kappa \text {-bdd }}$ holds iff there is a $\kappa$-bounded colouring $c$ of $\left[\omega_{1}\right]^{2}$ such that for each $A, \in\left[\omega_{1}\right]^{\omega}$ and $B \in\left[\omega_{1}\right]^{\omega_{1}}$ there is $\xi \in \operatorname{ran} c$ such that $|\{\{\alpha, \beta\} \in[A, B]: c(\alpha, \beta)=\xi\}|=k$.

Although an $\mathrm{AR}^{(k)}$-function establishes $\omega_{1} \nrightarrow^{*}\left[\left(\omega ; \omega_{1}\right)\right]_{k \text {-bdd }}$, there is no function which c.c.c.-indestructibly establishes $\omega_{1} \nrightarrow^{*}\left[\left(\omega ; \omega_{1}\right)\right]_{k \text {-bdd }}$ because Martin's Axiom implies $\omega_{1} \rightarrow^{*}\left[\left(\omega, \omega_{1}\right)\right]_{2 \text {-bdd }}$. Indeed, Martin's Axiom (even $\left.\mathfrak{p}>\omega_{1}\right)$ clearly implies $\omega_{1} \rightarrow\left[\left(\omega, \omega_{1}\right)\right]_{2}$, so it is enough to show that

$$
\omega_{1} \rightarrow\left[\left(\omega, \omega_{1}\right)\right]_{2} \text { implies } \omega_{1} \rightarrow^{*}\left[\left(\omega, \omega_{1}\right)\right]_{2 \text {-bdd }} .
$$

We prove $(\star)$ using an argument Galvin used to prove that $\lambda \rightarrow(\alpha)_{k}^{n}$ implies $\lambda \rightarrow^{*}(\alpha)_{k \text {-bdd }}^{n}$. Let $f:\left[\omega_{1}\right]^{2} \rightarrow \omega_{1}$ be 2 -bounded. Then there is a 
function $g:\left[\omega_{1}\right]^{2} \rightarrow 2$ such that $f(x)=f(y)$ implies $g(x) \neq g(y)$. Since $\omega_{1} \rightarrow\left[\left(\omega, \omega_{1}\right)\right]_{2}$ holds there are $A \in\left[\omega_{1}\right]^{\omega}$ and $B \in\left[\omega_{1}\right]^{\omega_{1}}$ such that $g^{\prime \prime}[A, B]=\{i\}$ for some $i<2$. Then $f \uparrow[A, B]$ is injective. So $(\star)$ holds.

ThEOREM 3.5. If GCH holds and $f:\left[\omega_{1}\right]^{2} \rightarrow \omega_{1}$ is an $A R^{(k)}$-function then there is a set $X \in\left[\omega_{1}\right]^{\omega_{1}}$ and a c.c.c. poset $Q$ such that

$V^{Q} \models X$ has a partition into countably many $f$-rainbow sets.

Before proving the theorems above we need to introduce some notions.

Given a set $x$ denote by $\mathrm{TC}(x)$ the transitive closure of $x$. Let $\kappa$ be a large enough regular cardinal $\left(\kappa=\left(2^{\omega_{1}}\right)^{+}\right.$works $)$. Put $H_{\kappa}=\{x:|\mathrm{TC}(x)|<\kappa\}$ and $\mathcal{H}_{\kappa}=\left\langle H_{\kappa}, \in, \prec\right\rangle$, where $\prec$ is a well-ordering of $H_{\kappa}$.

DeFinition 3.6.

(a) A sequence $\vec{N}=\left\langle N_{\alpha}: \alpha \in A\right\rangle$ of countable, elementary submodels of $\mathcal{H}_{\kappa}$ is called an $A$-chain iff $A \subset \omega_{1}$ and whenever $\alpha, \beta \in A$ with $\alpha<\beta$ we have $N_{\alpha} \in N_{\beta}$.

(b) Suppose that $\vec{N}=\left\langle N_{\alpha}: \alpha \in A\right\rangle$ is an $A$-chain and $Y \subset \omega_{1}$. We say that $Y$ is separated by $\vec{N}$ iff for each $C \in[Y]^{2}$ there is an $\alpha \in A$ with $\left|N_{\alpha} \cap C\right|=1$.

LEMma 3.7. Assume that $f$ is an $A R^{(k)}$-function. If $\left\langle N_{m}: m \leq n\right\rangle$ is an elementary $n+1$-chain, $f \in N_{0}, \vec{D}_{0}, \ldots, \vec{D}_{n-1} \in \mathbb{D}_{\omega}^{(k)}\left(\omega_{1}\right) \cap N_{0}$, and $\alpha_{m} \in N_{m+1} \backslash N_{m}$ for $m<n$ then the set

$$
\left\{i<\omega:(\forall m<n) \alpha_{m} \in \operatorname{Hom}\left(\vec{D}_{m}(i), f\right)\right\}
$$

is infinite.

Proof. We prove the lemma by induction on $n$. So assume that the set

$$
I=\left\{i<\omega:(\forall m<n-1) \alpha_{m} \in \operatorname{Hom}\left(\vec{D}_{m}(i), f\right)\right\}
$$

is infinite. (If $n=1$ then $I=\omega$.)

Write $I=\left\{i_{j}: j \in \omega\right\}$ and for each $\ell<\omega$ put

$$
\vec{E}^{\ell}=\left\langle\vec{D}_{n-1}\left(i_{j}\right): \ell \leq j<\omega\right\rangle .
$$

Let $\gamma_{\ell}$ be the minimal ordinal such that

$$
\omega_{1} \backslash \gamma_{\ell} \subset \bigcup\left\{\operatorname{Hom}\left(\vec{D}_{n-1}\left(i_{j}\right), f\right): j \in \omega \backslash \ell\right\} .
$$

Since $f$ is $\operatorname{AR}^{(k)}$ and $\vec{E}^{\ell} \in \mathbb{D}_{\omega}^{(k)}\left(\omega_{1}\right)$, we have $\gamma_{\ell}<\omega_{1}$. So if we take $\gamma=$ $\sup \left\{\gamma_{\ell}: \ell<\omega\right\}$ then for each $\alpha \in \omega_{1} \backslash \gamma$ the set

$$
J_{\alpha}=\left\{i \in I: \alpha \in \operatorname{Hom}\left(\vec{D}_{n-1}(i), f\right)\right\}
$$

is infinite.

Since $f, \vec{D}_{0}, \ldots, \vec{D}_{n-1}, \alpha_{0}, \ldots, \alpha_{n-2} \in N_{n-1}$ we have $I \in N_{n-1}$ and so $\vec{E}^{\ell} \in N_{n-1}$ as well. Thus $\left\langle\gamma_{\ell}: \ell<\omega\right\rangle \in N_{n-1}$ because it is definable there 
and so $\gamma=\sup \left\langle\gamma_{\ell}: \ell<\omega\right\rangle \in N_{n-1}$ as well. Hence $\alpha_{n-1} \in N_{n} \backslash N_{n-1} \subset \omega_{1} \backslash \gamma$ and so $J_{\alpha_{n-1}}$ is infinite.

But

$$
J_{\alpha_{n-1}}=\left\{i<\omega:(\forall m<n) \alpha_{m} \in \operatorname{Hom}\left(\vec{D}_{m}(i), f\right)\right\},
$$

so we are done.

Proof of Theorem 3.5. Let $\vec{N}=\left\langle N_{\xi}: \xi<\omega_{1}\right\rangle$ be an $\omega_{1}$-chain with $f \in N_{0}$ and let $X \in\left[\omega_{1}\right]^{\omega_{1}}$ be $\vec{N}$-separated. Let $H_{\omega_{1}}$ be the family of those sets whose transitive closure is countable.

Lemma 3.8. $H_{\omega_{1}} \subset \bigcup\left\{N_{\xi}: \xi<\omega_{1}\right\}$ under $C H$.

Proof. Since CH holds we have $\left|H_{\omega_{1}}\right|=\omega_{1}$. Since $N_{0}$ is an elementary submodel of $\mathcal{H}_{\kappa}$ the family $H_{\omega_{1}}$ has an enumeration $\vec{h}=\left\langle h_{\alpha}: \alpha<\omega_{1}\right\rangle \in N_{0}$. Then

(1) $\left\{h_{\alpha}: \alpha \in N_{\mu} \cap \omega_{1}\right\} \subset N_{\mu}$.

For $\nu<\mu<\omega_{1}$ we have $N_{\nu} \in N_{\mu}$ and so $N_{\nu} \cap \omega_{1} \in N_{\mu} \cap \mu$. Thus

(2) $\mu \subset N_{\mu} \cap \omega_{1}$ for $\mu<\omega_{1}$.

(1) and (2) together give the statement of the lemma.

Let us recall that $\operatorname{Fn}(X, \omega)$ denotes the family of finite functions mapping a subset of $X$ into $\omega$. Define the poset $\mathcal{Q}=\langle Q, \leq\rangle$ as follows:

$$
Q=\left\{q \in \operatorname{Fn}(X, \omega): q^{-1}\{n\} \text { is an } f \text {-rainbow for each } n \in \operatorname{ran} q\right\},
$$

and let $q \leq q^{\prime}$ iff $q \supset q^{\prime}$.

Lemma 3.9. $\mathcal{Q}$ satisfies c.c.c.

Proof. Assume that $\left\{q_{\nu}: \nu<\omega_{1}\right\} \subset Q$. Let $x_{\nu}=\operatorname{dom} q_{\nu}, L_{\nu}=\operatorname{ran} q_{\nu}$, and $x_{\nu, \ell}=q_{\nu}^{-1}\{\ell\}$ for $\ell \in L_{\nu}$. For two subsets $x$ and $y$ or ordinals we write $x<y$ iff $\sup (x)<\min (y)$.

We can assume that

(1) $\left\{x_{\nu}: \nu<\omega_{1}\right\}$ forms a $\Delta$-system with kernel $x$,

(2) $x<\left(x_{\zeta} \backslash x\right)<\left(x_{\xi} \backslash x\right)$ for $\zeta<\xi<\omega_{1}$,

(3) $L_{\nu}=L$ for each $\nu<\omega_{1}$,

(4) there is a $q$ such that $q_{\nu}\left\lceil[x]^{2}=q\right.$ for each $\nu<\omega_{1}$.

For $\zeta \in \omega_{1}$ let

$$
F(\zeta)=\left\{\xi<\omega_{1}: f^{\prime \prime}\left[x_{\zeta}, x_{\zeta} \backslash x\right] \cap f^{\prime \prime}\left[x_{\xi}, x_{\xi} \backslash x\right] \neq \emptyset\right\} .
$$

Since $f$ is $k$-bounded, $F(\zeta)$ is finite, and so there is an $F$-free set $Z=\left\{\zeta_{i}\right.$ : $\left.i<\omega_{1}\right\} \in\left[\omega_{1}\right]^{\omega_{1}}$, i.e. $\zeta_{j} \notin F\left(\zeta_{i}\right)$ for $i \neq j<\omega_{1}$.

For $x \in \omega_{1}$ let $\varrho(x)=\min \left\{\nu: x \in N_{\nu}\right\}$. For each $\xi \in X$ pick $d_{\xi} \in\left[\omega_{1}\right]^{k}$ such that $\xi \in d_{\xi}$ and $\varrho(\eta)=\varrho(\xi)$ for each $\eta \in d_{\xi}$. For $\zeta \in Z$ let $D_{\zeta}=$ $\left\{d_{\xi}: \xi \in x_{\zeta} \backslash x\right\}$. Let $\vec{D}=\left\langle D_{\zeta_{i}}: i<\omega\right\rangle$. Clearly $\vec{D} \in \mathbb{D}_{\omega}^{(k)}\left(\omega_{1}\right)$. Since 
CH holds there is $\gamma<\omega_{1}$ such that $\vec{D} \in N_{\gamma}$. Let $\zeta \in Z$ be such that $N_{\gamma} \cap\left(x_{\zeta} \backslash x\right)=\emptyset$.

Apply Lemma 3.7 for $n=\left|x_{\zeta} \backslash x\right|, \vec{D}_{m}=\vec{D}$ for $m<n$ and $\left\{\alpha_{m}: m<n\right\}$ $=x_{\zeta} \backslash x$. Then there is $i<\omega$ such that

$$
(\forall m<n) \alpha_{m} \in \operatorname{Hom}(\vec{D}(i), f) .
$$

By the construction it means that

$$
\left(\forall \eta \in x_{\zeta} \backslash x\right)\left(\forall \xi \in x_{\zeta_{i}} \backslash x\right)\left(\forall \delta \in d_{\xi}\right) f(\delta, \eta)=f(\xi, \eta) .
$$

Claim. $q_{\zeta_{i}} \cup q_{\zeta} \in Q$, i.e. $f$ is 1-1 on $\left[x_{\zeta_{i}, \ell} \cup x_{\zeta, \ell}\right]^{2}$ for all $\ell \in L$.

Let $\xi, \eta, \xi^{\prime}, \eta^{\prime} \in x_{\zeta_{i}, \ell} \cup x_{\zeta, \ell}$ with $\xi<\eta$ and $\xi^{\prime}<\eta^{\prime}$ such that $f(\xi, \eta)=$ $f\left(\xi^{\prime}, \eta^{\prime}\right)$.

Assume first that $\{\xi, \eta\},\left\{\xi^{\prime}, \eta^{\prime}\right\} \in\left[x_{\zeta_{i}, \ell}\right]^{2} \cup\left[x_{\zeta, \ell}\right]^{2}$. Since $q_{\zeta_{i}}, q_{\zeta} \in Q$ we can assume that $\{\xi, \eta\} \in\left[x_{\zeta_{i}, \ell}\right]^{2} \backslash\left[x_{\zeta, \ell}\right]^{2}$ and $\left\{\xi^{\prime}, \eta^{\prime}\right\} \in\left[x_{\zeta, \ell}\right]^{2} \backslash\left[x_{\zeta_{i}, \ell}\right]^{2}$ (or $f(\xi, \eta)=f\left(\xi^{\prime}, \eta^{\prime}\right)$ implies $\left.\{\xi, \eta\}=\left\{\xi^{\prime}, \eta^{\prime}\right\}\right)$. Then $f(\xi, \eta) \in f^{\prime \prime}\left[x_{\zeta_{i}}, x_{\zeta_{i}} \backslash x\right]$ and $f\left(\xi^{\prime}, \eta^{\prime}\right) \in f^{\prime \prime}\left[x_{\zeta}, x_{\zeta} \backslash x\right]$, so $\zeta_{i} \notin F(\zeta)$ implies $f(\xi, \eta) \neq f\left(\xi^{\prime}, \eta^{\prime}\right)$.

So we can assume that e.g. $\{\xi, \eta\} \notin\left[x_{\zeta_{i}, \ell}\right]^{2} \cup\left[x_{\zeta, \ell}\right]^{2}$, i.e. $\xi \in x_{\zeta_{i}, \ell} \backslash x$ and $\eta \in x_{\zeta, \ell} \backslash x$. But we know that

$$
\left(\forall \delta \in d_{\xi}\right) f(\delta, \eta)=f(\xi, \eta) .
$$

Since $f$ is $k$-bounded and $\left|d_{\xi}\right|=k$ we have

$$
\left\{\left\{\xi^{\prime}, \eta^{\prime}\right\}: f\left(\xi^{\prime}, \eta^{\prime}\right)=f(\xi, \eta)\right\}=\left\{\{\delta, \eta\}: \delta \in d_{\xi}\right\} .
$$

But $d_{\xi} \cap\left(x_{\zeta_{i}, \ell} \cup x_{\zeta, \ell}\right)=\{\xi\}$ because $\varrho(\delta)=\varrho(\xi)$ for each $\delta \in d_{\xi}$. Hence $f\left(\xi^{\prime}, \eta^{\prime}\right)=f(\xi, \eta)$ implies $\xi=\xi^{\prime}$ and $\eta=\eta^{\prime}$.

Since $\{q \in Q: \xi \in \operatorname{dom} q\}$ is dense in $\mathcal{Q}$ for each $\xi \in X$, we see that if $\mathcal{G}$ is the generic filter in $Q$ and $g=\bigcup \mathcal{G}$, then $\left\{g^{-1}\{n\}: n \in \omega\right\}$ is a partition of $X$ into countably many $f$-rainbow sets, which completes the proof of Theorem 3.5.

To prove Theorem 3.4 we need some more preparation. We will use a black box theorem from [11].

Given a set $K$ and a natural number $m$ let

$$
\operatorname{Fn}_{m}\left(\omega_{1}, K\right)=\left\{s: s \text { is a function, } \operatorname{dom}(s) \in\left[\omega_{1}\right]^{m}, \operatorname{ran}(s) \subset K\right\} .
$$

A sequence $\left\langle s_{\alpha}: \alpha<\omega_{1}\right\rangle \subset \operatorname{Fn}_{m}\left(\omega_{1}, K\right)$ is dom-disjoint iff $\operatorname{dom}\left(s_{\alpha}\right) \cap$ $\operatorname{dom}\left(s_{\beta}\right)=\emptyset$ all $\alpha<\beta<\omega_{1}$.

Let $H$ be a graph on $\omega_{1} \times K$ and $m \in \omega$. We say that $H$ is $m$-solid if given any dom-disjoint sequence $\left\langle s_{\alpha}: \alpha<\omega_{1}\right\rangle \subset \mathrm{Fn}_{m}\left(\omega_{1}, K\right)$ there are $\alpha<\beta<\omega_{1}$ such that

$$
\left[s_{\alpha}, s_{\beta}\right] \subset H .
$$

$H$ is called strongly solid iff it is $m$-solid for each $m \in \omega$. 
Black Box Theorem ([11, Theorem 2.2]). Assume $2^{\omega_{1}}=\omega_{2}$. If $H$ is a strongly solid graph on $\omega_{1} \times K$, where $|K| \leq 2^{\omega_{1}}$, then for each $m \in \omega$ there is a c.c.c. poset $P$ of size $\omega_{2}$ such that

$$
V^{P}=H \text { is c.c.c.-indestructibly } m \text {-solid. }
$$

The theorem above is build on a method of Abraham and Todorčević from $[2]$.

We need one more lemma before we can apply the Black Box Theorem above.

Lemma 3.10. There is a function $r: \omega_{1} \rightarrow \omega$ such that for any $A, B \in$ $\left[\omega_{1}\right]^{<\omega}$ if $r(A)=r(B)$ then $A \cap B$ is an initial segment of $A$ and $B$.

Proof. Let $\mathcal{D}$ be a countable dense subset of the product space $\omega^{\omega_{1}}$. Moreover, for each $\alpha<\omega_{1}$ fix a function $f_{\alpha}: \alpha \stackrel{1-1}{\longrightarrow} \omega$. Let $A=\left\{\alpha_{0}, \ldots, \alpha_{n-1}\right\}$ $\in\left[\omega_{1}\right]^{<\omega}$ with $\alpha_{0}<\cdots<\alpha_{n-1}$.

Pick $d_{A} \in \mathcal{D}$ such that $d_{A}\left(\alpha_{i}\right)=i$ for each $i<|A|$. Let

$$
r(A)=\left\langle d_{A},\left\langle f_{\alpha_{i}}^{\prime \prime}\left(A \cap \alpha_{i}\right): i<|A|\right\rangle\right\rangle .
$$

Since the range of $r$ is countable it is enough to prove that if $r(A)=r(B)$ then $A \cap B$ is an initial segment of $A$ and $B$.

Write $A=\left\{\alpha_{i}: i<m\right\}$ with $\alpha_{0}<\cdots<\alpha_{n-1}$, and $B=\left\{\beta_{j}: j<m\right\}$ with $\beta_{0}<\cdots<\beta_{m-1}$.

Assume that $\alpha_{i}=\beta_{j}$. Then $d_{A}\left(\alpha_{i}\right)=i$ and $d_{B}\left(\beta_{j}\right)=j$. Since $d_{A}=d_{B}$ it follows that $i=j$. So $r(A)=r(B)$ yields $f_{\alpha_{i}}^{\prime \prime}\left(A \cap \alpha_{i}\right)=f_{\alpha_{i}}^{\prime \prime}\left(B \cap \alpha_{i}\right)$. Since $f_{\alpha_{i}}$ is $1-1$ on $\alpha_{i}$ it follows that $A \cap \alpha_{i}=B \cap \alpha_{i}$.

We will use the following corollary of this lemma.

COROllary 3.11. There is a function $r: \omega_{1} \rightarrow \omega$ such that for any $A, B \in\left[\omega_{1}\right]^{<\omega}$ if $\min (A) \neq \min (B)$ and $r(A)=r(B)$ then $A \cap B=\emptyset$.

Proof of Theorem 3.4. Let $\vec{N}=\left\langle N_{\xi}: \xi<\omega_{1}\right\rangle$ be an $\omega_{1}$-chain with $f \in N_{0}$. Fix the function $r$ from Corollary 3.11 above. For $\xi \in \omega_{1}$ let $\varrho(\xi)=$ $\min \left\{\nu: \xi \in N_{\nu}\right\}$. Let $K=\left[\omega_{1}\right]^{k} \times \omega_{1} \times \omega$. For any function $c:\left[\omega_{1}\right]^{2} \rightarrow \omega_{1}$ define a graph $H_{c}$ on $\omega_{1} \times K$ as follows.

If $x, x^{\prime} \in \omega_{1} \times K, x=\langle\zeta,\langle d, \xi, m\rangle\rangle, x^{\prime}=\left\langle\zeta^{\prime},\left\langle d^{\prime}, \xi^{\prime}, m^{\prime}\right\rangle\right\rangle, \zeta<\zeta^{\prime}$, let $\left\{x, x^{\prime}\right\}$ be an edge in $H_{c}$ provided the conditions

(1) $\varrho(\xi)=\zeta$ and $\varrho\left(\xi^{\prime}\right)=\zeta^{\prime}$,

(2) $\zeta<\min d$ and $\zeta^{\prime}<\min d^{\prime}$,

(3) $r(\{\zeta\} \cup d)=m$ and $r\left(\left\{\zeta^{\prime}\right\} \cup d^{\prime}\right)=m^{\prime}$,

(4) $m=m^{\prime}$

imply

(5) $c\left(\delta, \xi^{\prime}\right)=c\left(\varepsilon, \xi^{\prime}\right)$ for all $\delta, \varepsilon \in d$. 
LEMMA 3.12. If $H_{c}$ is 1-solid for some colouring $c:\left[\omega_{1}\right]^{2} \rightarrow \omega_{1}$ then $c$

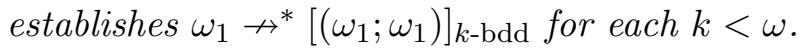

Proof. We will show that for all $X=\left\{\xi_{\beta}: \beta<\omega_{1}\right\} \in\left[\omega_{1}\right]^{\omega_{1}}$ and for any disjoint family $\left\{d_{\alpha}: \alpha<\omega_{1}\right\} \subset\left[\omega_{1}\right]^{k}$ there are $\alpha<\beta<\omega_{1}$ such that $\max d_{\alpha}<\xi_{\beta}$ and $\left|c^{\prime \prime}\left[d_{\alpha},\left\{\xi_{\beta}\right\}\right]\right|=1$.

By thinning out and renumbering the sequences we can assume that

- $\varrho\left(\xi_{\alpha}\right)<\min d_{\alpha}<\max d_{\alpha}<\varrho\left(\xi_{\beta}\right)$ for $\alpha<\beta<\omega_{1}$,

- $r\left(\left\{\varrho\left(\xi_{\alpha}\right) \cup d_{\alpha}\right\}\right)=m$ for some $m \in \omega$ for each $\alpha \in \omega_{1}$.

Let $x_{\alpha}=\left\langle\varrho\left(\xi_{\alpha}\right),\left\langle d_{\alpha}, \xi_{\alpha}, m\right\rangle\right\rangle$ for $\alpha<\omega_{1}$. Since the sequence $\left\langle\left\{x_{\alpha}\right\}: \alpha<\omega_{1}\right\rangle$ is dom-disjoint, and (1)-(4) hold for each $\alpha<\beta<\omega_{1}$, there are $\alpha<\beta<\omega_{1}$ such that (5) holds for $x_{\alpha}$ and $x_{\beta}$ because $H_{c}$ is 1 -solid, i.e. $\left|c^{\prime \prime}\left[d_{\alpha},\left\{\xi_{\beta}\right\}\right]\right|=1$, which was to be proved.

LEMMA 3.13. If $c$ is an $A R^{(k)}$-function and $C H$ holds then $H_{c}$ is strongly solid.

Proof. Let $m \in \omega$ and $\left\langle x_{\alpha}: \alpha<\omega_{1}\right\rangle \subset \operatorname{Fn}_{m}\left(\omega_{1}, K\right)$ be a dom-disjoint sequence. Write $x_{\alpha}=\left\{x_{\alpha, i}: i<m\right\}, x_{\alpha, i}=\left\langle\zeta_{\alpha, i},\left\langle d_{\alpha, i}, \xi_{\alpha, i}, n_{\alpha, i}\right\rangle\right\rangle$. A pair $\langle\zeta,\langle d, \xi, m\rangle\rangle \in \omega_{1} \times K$ is good iff

(g1) $\varrho(\xi)=\zeta$

(g2) $\zeta<\min d$

(g3) $r(\{\zeta\} \cup d)=m$.

We can assume that every $x_{\alpha, i}$ is good because if $\langle\zeta, t\rangle \in \omega_{1} \times K$ is not good then $\left\{\langle\zeta, t\rangle,\left\langle\zeta^{\prime}, t^{\prime}\right\rangle\right\} \in H_{c}$ for each $\left\langle\zeta^{\prime}, t^{\prime}\right\rangle \in \omega_{1} \times K$ with $\zeta^{\prime} \neq \zeta$. So we have

(i) $\varrho\left(\xi_{\alpha, i}\right)=\zeta_{\alpha, i}$,

(ii) $\zeta_{\alpha, i}<\min d_{\alpha, i}$,

(iii) $r\left(\left\{\zeta_{\alpha, i}\right\} \cup\left(d_{\alpha, i}\right)\right)=n_{i}$.

By thinning out our sequence we can assume that

(iv) $n_{\alpha, i}=n_{i}$

(v) $\max d_{\alpha, i}<\zeta_{\beta, j}$ for $\alpha<\beta<\omega_{1}$ and $i, j<m$.

Let $N=\left\{n_{i}: i<m\right\}$. For $\alpha<\omega_{1}$ and $n \in N$ put $D_{\alpha, n}=\left\{d_{\alpha, i}: n_{i}=n\right\}$.

Claim. $D_{\alpha, n} \in \mathcal{D}^{(k)}\left(\omega_{1}\right)$.

Indeed, if $i \neq j<m$ and $n_{i}=n_{j}$ then $r\left(\left\{\zeta_{\alpha, i}\right\} \cup d_{\alpha, i}\right)=n_{i}=n_{j}=$ $r\left(\left\{\zeta_{\alpha, j}\right\} \cup d_{\alpha, j}\right)$ but $\min \left(\left\{\zeta_{\alpha, i}\right\} \cup d_{\alpha, i}\right)=\zeta_{\alpha, i} \neq \zeta_{\alpha, j}=\min \left(\left\{\zeta_{\alpha, j}\right\} \cup d_{\alpha, j}\right)$ so $d_{\alpha, i} \cap d_{\alpha, j}=\emptyset$ by the choice of the function $r$.

(iii) and (v) together give $\max \left(\bigcup D_{\alpha, n}\right)<\min \left(\bigcup D_{\beta, n}\right)$ for $\alpha<\beta<\omega_{1}$ and $n \in N$. Thus $\vec{D}_{n}^{\prime}=\left\langle D_{\ell, n}: \ell<\omega\right\rangle \in \mathbb{D}_{\omega}^{(k)}\left(\omega_{1}\right)$. 
Since CH holds, from Lemma 3.8 it follows that there is $\gamma<\omega_{1}$ such that $\left\{\vec{D}_{n}^{\prime}: n \in N\right\} \subset N_{\gamma}$. Pick $\alpha<\omega_{1}$ such that $N_{\gamma} \cap\left\{\zeta_{\alpha, j}: j<m\right\}=\emptyset$. Let $\vec{D}_{j}=\vec{D}_{n_{j}}^{\prime}$ for $j<m$.

We are going to apply Lemma 3.7 as follows: $\vec{M}=\left\langle N_{\gamma}, N_{\zeta_{j}}: j\langle m\rangle\right.$ is an elementary $m+1$-chain, $f, \vec{D}_{0}, \ldots, \vec{D}_{m-1} \in N_{0}$ and $\xi_{\alpha, j} \in N_{\zeta_{j}} \backslash N_{\zeta_{j-1}}$ for $j<m$, where $\zeta_{-1}=\gamma$. Hence, by Lemma 3.7 there is $\ell<\omega$ such that for each $j<m$,

$$
\xi_{\alpha, j} \in \operatorname{Hom}\left(\vec{D}_{j}(\ell), f\right) .
$$

Claim. $\left[x_{\ell}, x_{\alpha}\right] \subset H_{c}$.

Let $i, j<m$. We show $\left\{x_{\ell, i}, x_{\alpha, j}\right\} \in H_{c}$. (2)-(4) hold by construction. If $n_{i} \neq n_{j}$ then (1) fails so we are done. Assume that $n_{i}=n_{j}=n \in N$. Then $d_{\ell, i} \in \vec{D}_{n}^{\prime}(\ell)=\vec{D}_{j}(\ell)$. Thus

$$
\left(\forall \delta, \delta^{\prime} \in d_{\ell, i}\right) f\left(\delta, \xi_{\alpha, j}\right)=f\left(\delta^{\prime}, \xi_{\alpha, j}\right)
$$

by (०). Hence (5) holds and so $\left\{x_{\ell, i}, x_{\alpha, j}\right\} \in H_{c}$.

Now we can easily conclude the proof of 3.4.

Let $f:\left[\omega_{1}\right]^{2} \rightarrow \omega_{1}$ be an $\mathrm{AR}^{(k)}$-function. By Lemma 3.13, the graph $H_{f}$ is strongly solid. Since GCH holds, we can apply our Black Box Theorem to find a c.c.c. poset $P$ such that

$$
V^{P} \models H_{f} \text { is c.c.c.-indestructibly } 1 \text {-solid. }
$$

But then, by Lemma 3.12,

$$
V^{P} \models f \text { c.c.c.-indestructibly establishes } \omega_{1} \nrightarrow^{*}\left[\left(\omega_{1} ; \omega_{1}\right)\right]_{k \text {-bdd }} \text {. }
$$

Proof of Theorem 1.2. Since GCH holds, by Lemma 3.3 there is an $\mathrm{AR}^{(k)}$-function $g:\left[\omega_{1}\right]^{2} \rightarrow \omega_{1}$. By Theorem 3.5 there is a set $X \in\left[\omega_{1}\right]^{\omega_{1}}$ and a c.c.c. poset $Q$ such that

$V^{Q} \models X$ has a partition into countably many $g$-rainbow sets.

Let $h: \omega_{1} \rightarrow X$ be a bijection and put $f=g \circ h$. Then

$V^{Q} \models \omega_{1}$ has a partition into countably many $f$-rainbow sets.

Since $f$ is an $\mathrm{AR}^{(k)}$-function as well, we can apply Theorem 3.4 to deduce that

$$
V^{P} \models f \text { c.c.c.-indestructibly establishes } \omega_{1} \nrightarrow^{*}\left[\left(\omega_{1} ; \omega_{1}\right)\right]_{k \text {-bdd }}
$$

for some c.c.c. poset $P$, which proves the theorem.

Lemma 3.3 and Theorem 3.4 give immediately

Corollary 3.14. $\omega_{1} \nrightarrow^{*}\left[\left(\omega_{1} ; \omega_{1}\right)\right]_{k \text {-bdd }}$ is consistent with Martin's Axiom for each natural number $k$. 
Acknowledgements. We would like to thank the referee for his helpful suggestions and comments.

\section{References}

[1] U. Abraham, J. Cummings and C. Smyth, Some results in polychromatic Ramsey theory, J. Symbolic Logic 72 (2007), 865-896.

[2] U. Abraham and S. Todorčević, Martin's Axiom and first countable S-and L-spaces, in: Handbook of Set-Theoretic Topology, K. Kunen and J. E. Vaughan (eds.), NorthHolland, New York, 1984, 327-345.

[3] J. E. Baumgartner, Results and independence proofs in combinatorial set theory, Ph.D. Thesis, Univ. of California, Berkeley, 1970.

[4] J. Baumgartner and A. Hajnal, A proof (involving Martin's axiom) of a partition relation, Fund. Math. 78 (1973), 193-203.

[5] P. Erdős and A. Hajnal, Unsolved and solved problems in set theory, in: Proc. Tarski Symposium (Berkeley, 1971), Proc. Sympos. Pure Math. 25, Amer. Math. Soc., 1974, 269-287.

[6] —, - Embedding theorems for graphs establishing negative partition relations, Period. Math. Hungar. 9 (1978), 205-230.

[7] F. Galvin, letter to S. Todorčević.

[8] A. Hajnal, unpublished note.

[9] —, Rainbow Ramsey theorems for colorings establishing negative partition relations, Fund. Math. 198 (2008), 255-262.

[10] S. Shelah, Coloring without triangles and partition relations, Israel J. Math. 20 (1975), 1-12.

[11] L. Soukup, Indestructible properties of S-and L-spaces, Topology Appl. 112 (2001), $245-257$.

[12] S. Todorčević, Forcing positive partition relations, Trans. Amer. Math. Soc. 280 (1983), 703-720.

Alfréd Rényi Institute of Mathematics

V. Reáltanoda utca 13-15

H-1053 Budapest, Hungary

E-mail: soukup@renyi.hu

Received 28 April 2008;

in revised form 20 July 2008 